\title{
The role of quantitative changes in the epxression of insulin receptor substrate-1 and nuclear ubiquitin in abnormal glycometabolism in the livers of KKay mice and the relative therapeutic mechanisms of Astragalus polysaccharide
}

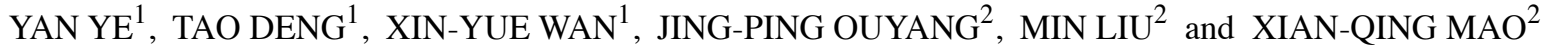 \\ ${ }^{1}$ Department of Gastroenterology, Renmin Hospital of Wuhan University, Wuhan, Hubei 430060; \\ ${ }^{2}$ School of Basic Medical Sciences, Wuhan University, Wuhan, Hubei 430071, P.R. China
}

Received August 19, 2013; Accepted November 26, 2013

DOI: $10.3892 /$ ijmm.2013.1580

\begin{abstract}
Ubiquitin and the ubiquitination pathway are important regulators of insulin signaling. The insulin receptor substrate-1 (IRS-1), an ubiquitin-interacting adaptor protein, serves as the key docking protein in insulin signaling. The effects of this dynamic interaction and the changes in ubiquitin expression on hepatic insulin signaling, as well as the relative therapeutic effects of Astragalus polysaccharide (APS) have not yet been elucidated. In this study, we aimed to investigate the abnormal changes which occur in the levels of IRS-1 and ubiquitin in the livers of mice (mice with insulin resistance and diabetes), and to elucidate the possible mechanisms responsible for these changes. A control group (CG), an insulin resistance group (IG) and a diabetes group (DG) were respectively composed of 12-week-old C57BL/6J mice fed a normal diet, C57BL/6J mice fed a high-fat diet and KKay mice fed a high-fat diet, and treatment groups were composed of corresponding groups treated with APS $(\mathrm{CG}+\mathrm{A}, \mathrm{IG}+\mathrm{A}, \mathrm{DG}+\mathrm{A})$. All the mice were age-matched and grouped at random. After eight weeks, the mouse models were successfully established and the related physiological or biochemical indexes were detected using corresponding methods. Ubiquitin expression in the liver was detected by immunohistochemisty, and western blot analysis was used to detect the expression of IRS-1 and ubiquitin. The results revealed that the expression of IRS-1 in the DG was significantly lower compared to that in the CG and IG; however, the nuclear expression of ubiquitin and the ubiquitination levels of IRS-1, including body weight and blood glucose and triglyceride levels in the DG were significantly higher compared to those in the CG or IG $(\mathrm{P}<0.05)$. There
\end{abstract}

Correspondence to: Dr Tao Deng, Department of Gastroenterology, Renmin Hospital of Wuhan University, 238 Jiefang Road, Wuhan, Hubei 430060, P.R. China

E-mail: dengtao1120@hotmail.com

Key words: insulin receptor substrate-1, ubiquitination, Astragalus polysaccharide, glycometabolism was a significant improvement in the ubiquitination levels in $\mathrm{DG}+\mathrm{A}$, including the blood glucose and triglyceride levels compared with the DG $(\mathrm{P}<0.05)$. From the stage of insulin resistance to the stage of diabetes, the reduced expression of IRS-1 and its enhanced ubiquitination levels combined with the overexpression of nuclear ubiquitin contributed to the abnormal glycometabolism and the disruption of insulin signaling. APS showed beneficial effects, such as lowering body weight, as well as blood glucose and triglyceride levels, and these effects correlated with the downregulation of the ubiquitination levels of IRS-1 and the nuclear expression of ubiquitin.

\section{Introduction}

The insulin receptor substrate (IRS) protein family plays a crucial role in the insulin signaling cascade and the insulinlike growth factor (IGF) signal transduction pathway $(1,2)$. The importance of the IRS-1 isoform, one of four IRS family proteins, has been established by experiments on metabolic signaling pathways (3). As the first downstream element from the insulin/IGF signaling pathway, IRS-1 acts as a key mediator. There are several critical tyrosine residues that must be phosphorylated for the protein to become fully activated and continue the insulin/IGF signaling cascades. Previous studies have demonstrated the functions and bioactivities of the phosphorylation of IRS-1 (4,5). However, perhaps there are other important unknown facts about IRS-1 to be investigated from another research perspective. A previous study revealed that the degradation of IRS-1 is attributed to the role of the ubiquitin-proteasome system; this discovery was awarded the Nobel Prize in 2004 (6). By analyzing the ubiquitination levels of IRS-1 and the changes in ubiquitin expression which occur in vivo from the stage of insulin resistance to the stage of type 2 diabetes, novel insight may be acquired into the role of IRS-1.

Astragalus polysaccharide (APS) is a water-soluble heteroglycan, which is extracted, concentrated and purified from the dry root of Astragalus membranaceus, a Chinese medicinal herb. Related studies have revealed that APS exerts various therapeutic effects, such as improving a number of diabetesrelated disorders $(7,8)$ and regulating immune response $(9,10)$. It is worth noting that APS has shown various beneficial 
effects, such as improving glycometabolism and lipid metabolism $(11,12)$. Since it has been well established that insulin and the IGF signaling pathway exert various effects, particularly on metabolic function, it is worthwhile to investigate the regulatory mechanisms of APS and its effects on the insulin and IGF signaling pathway.

In this study, we aimed to investigate the abnormal changes which occur in the levels of IRS-1 and ubiquitin in the livers of mice (mice with insulin resistance and diabetes), and to elucidate the possible mechanisms responsible for these changes. We also aimed to determine the effects of these changes and to explore the related therapeutic mechanisms of APS on the expression of IRS-1 and the ubiquitination levels in the liver in vivo from the stage of insulin resistance to the stage of type 2 diabetes.

\section{Materials and methods}

Preparation, grouping and treatment of experimental animal models. Eight-week-old female C57BL/6J mice and eightweek-old female KKay mice were purchased from the Institute of Laboratory Animal Science, Chinese Academy of Medical Sciences (CAMS) and Peking Union Medical College (PUMC) [Experimental animal production license number: SCXK (Bj2003-0003)]. All animal experiments were performed under the approved protocols of the Animal Care and Use Committee of Wuhan University, Wuhan, China. All the mice were raised in the Animal Experimental Center of Wuhan University (Animal Biosafety Level III Laboratory). The animals were kept in a temperature controlled enviroment $\left(22-25^{\circ} \mathrm{C}\right)$ with a relative humidity of $60 \%$ in a 12 -h light/dark cycle. All the mice were provided access to food and water ad libitum. The $\mathrm{C} 57 \mathrm{BL} / 6 \mathrm{~J}$ mice were divided into two groups at random: one group was fed a normal diet [the constituents measured with the percentage of total calories ( $\mathrm{kcal}$ ) were $12 \%$ fat, $60 \%$ carbohydrate and $28 \%$ protein], and the other group was fed a high-fat diet (the constituents were $41 \%$ fat, $41 \%$ carbohydrate and $18 \%$ protein). The KKay mice were fed a high-fat diet. All the forage (the mouse diets) was sterilized with radiation by cobalt- 60 and vacuum-packed. After 4 weeks, [mice were 12 weeks old (referred to as the 12th week)] and at the end of the treatment period [mice were 20 weeks old (referred to as the 20th week)] blood glucose levels were measured using a OneTouch Ultra fast blood glucose meter (LifeScan, Inc., Milpitas, CA, USA). KKay mice with fasting blood glucose (FBG) levels $>12 \mathrm{mmol} / 1$ were diagnosed with diabetes. C57BL/6J mice (now 12 weeks old) fed a normal diet were subdivided into the control group (CG) and normal group treated with APS $(\mathrm{CG}+\mathrm{A})$ at random; the $\mathrm{C} 57 \mathrm{BL} / 6 \mathrm{~J}$ mice fed a high-fat diet were subdivided into the insulin resistance group (IG) and the insulin resistance group treated with APS $(\mathrm{IG}+\mathrm{A})$ at random; the KKay mice were subdivided into the diabetes group (DG) and the group treated with APS $(\mathrm{DG}+\mathrm{A})$ at random. The mice treated with APS were intragastrically administered APS at a dose of $700 \mathrm{mg} /$ $\mathrm{kg} /$ day for eight weeks; the mice not treated with APS were intragastrically administered the same volume of physiological saline per day. During the eight weeks of treatment, the general health status, body weight, as well as blood glucose, triglyceride and plasma insulin levels were monitored and measured each weekend. After the treatment was completed, all the mice were sacrificed (at 20 weeks of age; referred to as 20 th week) and the tissues were removed and collected. Some tissues were fixed in $10 \%$ formalin, while others were stored in liquid nitrogen.

Reagents and preparation of APS. Polyclonal antibody to IRS-1 and monoclonal antibody to ubiquitin were purchased from Cell Signaling Technology, Inc. (Danvers, MA, USA). Monoclonal antibody to GAPDH was purchased from Abcam, Inc. (Cambridge, MA, USA). The BCA protein quantification kit and Restore Western Blot Stripping Buffer were purchased from Pierce Biotechnology, Inc. (Rockford, IL, USA). ECL Western Blotting Detection Reagents were purchased from Kirkegaard \& Perry Laboratories, Inc. (Gaithersburg, MD, USA). The histostain-SP kit and DAB Horseradish Peroxidase Color Development kit were purchased from Beijing Zhongshan Golden Bridge Biotechnology Co., Ltd. (Beijing, China). Astragalus membranaceus was purchased from Shanghai Traditional Chinese Medicine Co., Ltd. (Shanghai, China) and identified by Hubei University of Chinese Medicine. APS was extracted from Astragalus membranaceus using the water decoction extraction process and purified with Sephadex G-150 column chromatography.

General health status and body weight of animals. The mental state, the sheen of coat and hair and the activity status of all the mice were observed daily, and the body weight of all the mice was measured weekly using an electronic scale.

Measurement of blood glucose levels. FBG and postprandial blood glucose (PBG) levels were measured weekly in blood obtained from the vena caudalis using a OneTouch Ultra fast blood glucose meter.

Measurement of plasma insulin levels. Plasma insulin levels were measured using a Mouse Insulin ELISA kit (Linco Research Inc., St. Charles, MO, USA). The operational principle of this kit is the analysis of antibodies conjugated to magnetic beads and double antibody sandwich ELISA. The plasma insulin levels of the control mice acted as the controls and all procedures were carried out in accordance with the manufacturer's instructions.

Measurement of blood triglyceride levels. After a 10-h fasting period, blood was taken from the heart of all the mice and serum was extracted from the blood using a hydroextractor. The triglyceride levels in the serum were measured using a triglyceride assay kit based on the GPO-PAP method (BioSino Bio-Technology and Science Inc., Beijing, China).

Measurement of hepatic triglyceride levels. The liver tissue was rinsed several times with physiological saline. The tissue was then cut into small sections and dried to constant weight. The dry tissue was worn to a powder and soaked in a solution of $0.2 \% \mathrm{NaCl}$. The suspension was then transferred into a glass homogenizer and homogenized for $30 \mathrm{sec}$ with a mixture of chloroform:formaldehyde $(2: 1)$. The intermixture was transferred into a tap funnel for phase separation. The organic phase was transferred into a rotary evaporator and evaporated till dry. Hepatic triglyceride levels were measured in the final product using the triglyceride assay kit. 
Immunohistochemisty. The liver tissue was rinsed with physiological saline, fixed in formaldehyde and embedded in paraffin wax. Sections of $5 \mu \mathrm{m}$ thickness were affixed to slides, deparaffinized and dehydrated, then the sections underwent blocking of endogenous peroxidase, antigen retrieval, serum blocking, incubation with primary antibody to ubiquitin, incubation with biotin-labeled secondary antibody, incubation with horseradish peroxidase-labeled streptavidin solution, staining with DAB solution, hematoxylin counterstaining and finally, dehydration, hyalinization, desiccation and mounting for observation under an optical microscope. Images were acquired to record changes.

Western blot analysis. A total of $50 \mathrm{mg}$ of liver tissue was placed in $200 \mu \mathrm{l}$ lysate and the tissue was ground to obtain homogenate using a homogenizer on ice. The homogenate was centrifuged at $4^{\circ} \mathrm{C}$ for $30 \mathrm{~min}$ and supernatant was obtained. Two microliters from the supernatant were added to the solution of the BCA kit and quantified for the content of total protein by ELISA (VA 200; Tecan, Salzburg, Austria); the remaining supernatant was mixed with $2 \mathrm{X}$ sample buffer and the mixture was boiled for $5 \mathrm{~min}$. Following electrophoresis transfer onto a PVDF membrane, the membrane was successively incubated with confining liquid, the diluted antibody of IRS-1 and the diluted secondary antibody. The membrane was then incubated with chemoluminescence solution for $1 \mathrm{~min}$ and covered with $\mathrm{X}$-ray film in a dark room. The dark bands on the X-ray film were scanned and transformed into images using a gel imaging system (Vilber Lourmat, Marne-la-Vallée, France). The images were used for quantitative analysis with image analysis Quantity One software (Bio-Rad, Hercules, CA, USA) and the color concentration of the dark bands was represented with the average grey density. After the detection of IRS-1, the membrane where the antibody of IRS-1 and the secondary antibody remained was incubated with the Restore Western Blot Stripping Buffer (Pierce Biotechnology, Inc.) for $1 \mathrm{~h}$ at room temperature. The blots were then stripped and the membrane was treated with blocking buffer. In order to measure the ubiquitination level of IRS-1, the same membrane was reprobed with the primary antibody to ubiquitin, as well as the primary antibody to GAPDH to confirm equal loading; thus, the relative value of grey density for ubiquitin to IRS-1 on the same membrane represented the ubiquitination level of IRS-1.

Statistical analysis. All the measurement data are presented as the means + SEM. A comparison among multiple groups was carried out by one-way analysis of variance and a comparison between two groups was carried out using Fisher's LSD method. Enumeration data from the results of immunohistochemisty were derived using the $\chi^{2}$ test. SPSS 19.0 software was used for statistical analysis and a value of $\mathrm{P}<0.05$ was considered to indicate a statistically significant difference.

\section{Results}

General health status of mice. The CG mice had a good mental state and were highly active; the DG mice were constantly in a crouched position and were generally sleepy; DG $+\mathrm{A}$ and $\mathrm{IG}+\mathrm{A}$ mice were in good spirits and had shiny coat and hair and were highly active.
Effect of quantitative changes in the expression of IRS-1 and nuclear ubiquitin

Comparison of body weight, blood glucose and plasma insulin levels, as well as triglyceride levels in blood and liver among $C G, I G$ and DG mice. On the 20th week, the body weight of the IG and DG mice was significantly increased compared with the CG mice (Fig. 1A). On the 12th week, PBG levels of the IG mice were not significantly higher compared to those of the CG mice (Fig. 1D); however, plasma insulin levels of the IG mice were significantly higher compared to those of the CG mice (Fig. 1B). These data indicated that the IG mice began to exhibit insulin resistance. On the 20th week, the PBG levels and plasma insulin levels of the IG mice were significantly higher than those of the CG mice (Fig. 1B and D), indicating that the insulin resistance of the IG mice had worsened. On the 20th week, the FBG levels $(12.4 \pm 0.9 \mathrm{mmol} / \mathrm{l})$ and PBG levels $(33.1 \pm 2.0 \mathrm{mmol} / \mathrm{l})$ of the DG mice were significantly higher compared to those of the $\mathrm{CG}$ mice; the plasma insulin levels were also higher in the DG mice (Fig. $1 \mathrm{~B}$ and D), indicating that the DG mice presented with symptoms of diabetes. On the 20th week, the blood triglyceride levels of the IG and DG mice were significantly higher compared to those of the CG mice and the hepatic triglyceride levels of the DG mice were also significantly increased compared to those of the CG mice (Fig. 1E and F).

Immunohistochemisty. As observed under a light microscope, the liver tissue of the IG mice showed partial cellular edema and steatosis, and ubiquitin was highly expressed, particularly in the nucleolus (Fig. 2B); the liver tissue of the DG mice showed severe steatosis, vacuolization and bridging necrosis, and ubiquitin was also highly expressed in the nucleolus (Fig. 2C).

Twelve fields of vision with high power lens (20x10 times) were selected at random for counting. A total of 400 hepatocytes were continuously counted on each field so that the percentage of positively stained cells and the staining intensity could be acquired and the synthetic score could be calculated. The letter ' $a$ ' represented the percentage of positively stained cells (no positively stained cells, $0 ; 1-10 \%$ positively stained cells, 1 ; 11-50, 2; 51-80\%, 3; 81-100\%, 4), the letter ' $b$ ' represented the staining intensity [negative staining (-), 0; weakly positive staining $(+), 1$; moderately positive staining $(++), 2$; strong staining $(+++), 3$ ]; the product of 'a' and 'b' represented the immunohistochemical score of the sample. An immunohistochemical score $\leq 2$ represented negative results, and a score of $\geq 3$ and $\leq 12$ represented positive results (Table I). Differential levels of nuclear ubiquitin expression were detected in the IG and DG mice; however, the CG mice showed negative results for the expression of ubiquitin in the nucleolus (Table II).

Western blot analysis. The expression of IRS-1 in the DG mice was significantly lower than that in the CG and IG mice; however, the ubiquitination level of the DG mice was significantly higher than that in the IG mice $(\mathrm{P}<0.05)$. There was no significant difference between the CG and IG mice as regards the expression of IRS-1 and its ubiquitination level $(\mathrm{P}>0.05)$ (Fig. 3).

\section{Effect of APS on insulin resistance \\ Comparison of physiological and biochemical indexes among groups. Our data demonstrated that on the 20th week,}



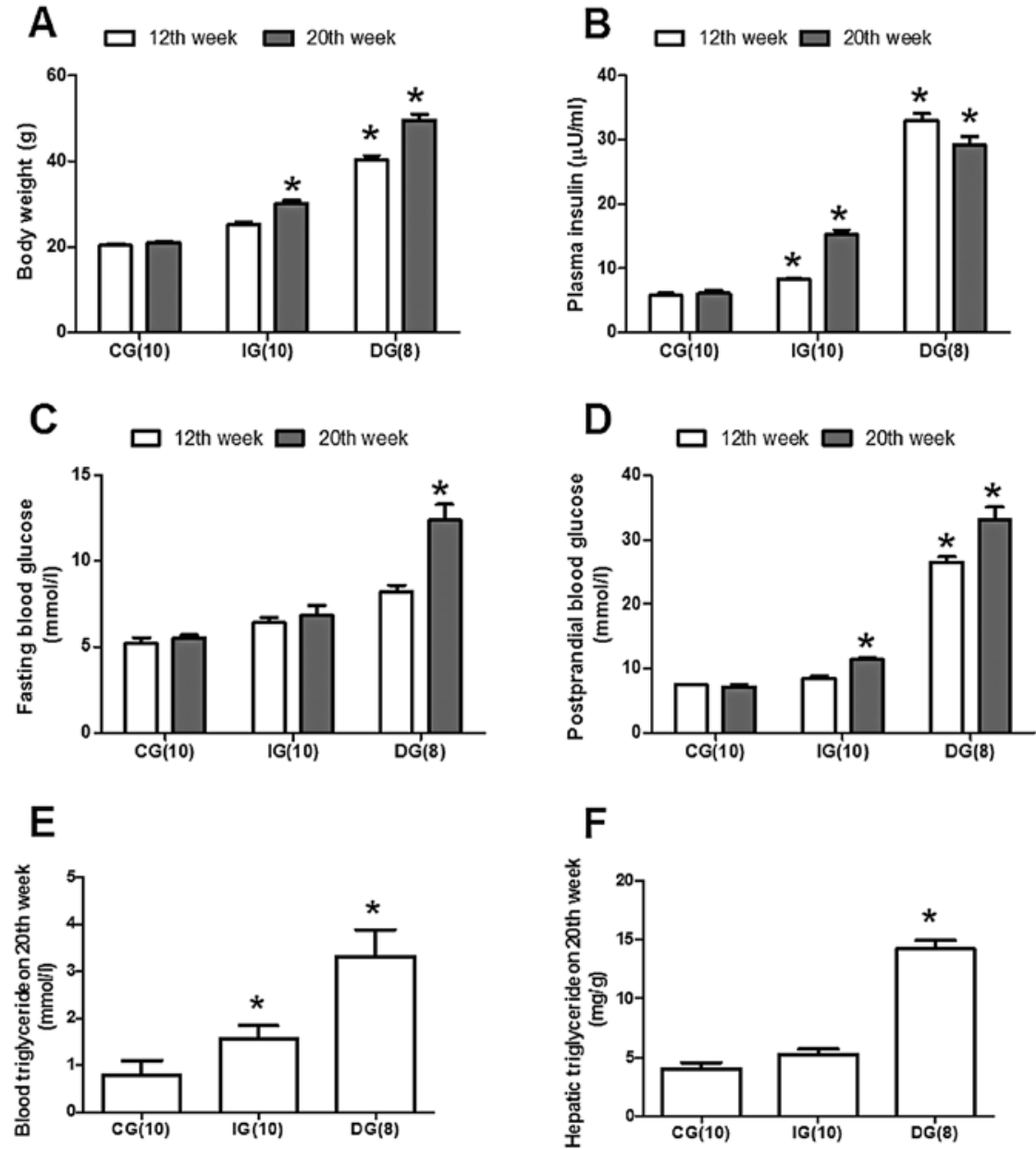

Figure 1. Comparison of (A) body weight, (B) plasma insulin levels, (C) fasting blood glucose levels, (D) postprandial blood glucose levels, (E) blood triglyceride levels, and (F) hepatic triglyceride levels among the different groups. CG, control group; IG, insulin resistance group; DG, diabetes group. Data are presented as the means \pm SEM. Numbers in brackets denote the number of animals studied. "P $<0.05$ or $0.01, \mathrm{CG}$ vs. the other groups on the same week.

Table I. IHC of nuclear ubiquitin expression in the different groups.

IHS score in each group

\begin{tabular}{lccrcrc} 
Field of \begin{tabular}{l} 
vision \\
\cline { 2 - 6 }
\end{tabular} & $\mathrm{CG}$ & $\mathrm{CG}+\mathrm{A}$ & $\mathrm{IG}$ & $\mathrm{IG}+\mathrm{A}$ & $\mathrm{DG}$ & $\mathrm{DG}+\mathrm{A}$ \\
\hline 1 & 0 & 0 & 12 & 2 & 12 & 3 \\
2 & 0 & 0 & 8 & 1 & 4 & 2 \\
3 & 0 & 0 & 12 & 2 & 12 & 4 \\
4 & 0 & 0 & 12 & 1 & 2 & 2 \\
5 & 0 & 0 & 6 & 1 & 12 & 0 \\
6 & 0 & 0 & 12 & 0 & 12 & 12 \\
7 & 0 & 0 & 2 & 6 & 2 & 2 \\
8 & 0 & 0 & 5 & 1 & 9 & 1 \\
9 & 0 & 0 & 9 & 0 & 1 & 2 \\
10 & 0 & 0 & 1 & 2 & 10 & 0 \\
11 & 0 & 0 & 10 & 5 & 2 & 2 \\
12 & 0 & 0 & 2 & 1 & 6 & 1
\end{tabular}

IHS, immunohistochemistry; CG, control group; IG, insulin resistance group; DG, diabetes group; $\mathrm{CG}+\mathrm{A}$, control group treated with Astragalus polysaccharide (A); IG + A, insulin resistance group treated with $\mathrm{A} ; \mathrm{DG}+\mathrm{A}$, diabetes group treated with A. An immunohistochemical score $\leq 2$ represented negative results, and a score of $\geq 3$ and $\leq 12$ represented positive results.
APS significantly reduced the weight of the IG mice, and decreased the PBG and plasma insulin levels in the IG mice compared with the $\mathrm{IG}+\mathrm{A}$ mice $(\mathrm{P}<0.05)$ (Fig. 4A, B and D), however, the plasma insulin levels of the IG + A mice were higher than those of the CG mice on the 20th week $(\mathrm{P}<0.05)$ (Fig. 4B).

Immunohistochemisty. As observed under a light microscope, cellular edema was markedly improved and the expression of ubiquitin in the nucleolus was greatly reduced in the $\mathrm{IG}+\mathrm{A}$ mice compared with the IG mice (Fig. $2 \mathrm{~B}$ and $\mathrm{E}$ ); however, the overall expression of ubiquitin in the $\mathrm{IG}+\mathrm{A}$ mice was higher than that in the $\mathrm{CG}$ and $\mathrm{CG}+\mathrm{A}$ mice (Fig. 2A, $\mathrm{D}$ and $\mathrm{E})$. The comparison of the results from immunohistochemisty among the different groups demonstrated that the expression of ubiquitin in the nucleolus of the IG mice was significantly higher than that in the $C G$ mice $(\mathrm{P}<0.05)$; however, this increase in expression was reversed by treatment with APS (Table II).

Western blot analysis. Both the $\mathrm{CG}+\mathrm{A}$ and $\mathrm{IG}+\mathrm{A}$ mice showed an upregulation in the expression of IRS-1, respectively compared with the CG and IG mice; however, there was no significant difference as regards the expression of IRS-1 and the ubiquitination level of IRS-1 among the different groups (Fig. 5). 
Table II. Comparison of the results of immunohistochemistry among the groups.

\begin{tabular}{|c|c|c|c|c|c|c|c|c|c|c|c|c|}
\hline & \multicolumn{12}{|c|}{ Group } \\
\hline & \multicolumn{2}{|c|}{$\mathrm{CG}$} & \multicolumn{2}{|c|}{$\mathrm{CG}+\mathrm{A}$} & \multicolumn{2}{|c|}{ IG } & \multicolumn{2}{|c|}{$\mathrm{IG}+\mathrm{A}$} & \multicolumn{2}{|c|}{ DG } & \multicolumn{2}{|c|}{$\mathrm{DG}+\mathrm{A}$} \\
\hline & + & - & + & - & + & - & + & - & + & - & + & - \\
\hline IHS score & 0 & 12 & 0 & 12 & $9^{a}$ & 3 & $2^{\mathrm{b}}$ & 10 & $8^{a}$ & 4 & $3^{c}$ & 9 \\
\hline
\end{tabular}

IHS, immunohistochemistry; CG, control group; IG, insulin resistance group; DG, diabetes group; CG + A, control group treated with Astragalus polysaccharide (A); IG + A, insulin resistance group treated with A; DG + A, diabetes group treated with A; +, positive staining; -, negative staining. Numbers indicate IHS score for positively and negatively stained cells. ${ }^{a} \mathrm{P}<0.05, \mathrm{CG}$ vs. the other groups; ${ }^{\mathrm{b}} \mathrm{P}<0.05, \mathrm{IG}+\mathrm{A}$ vs. IG; ${ }^{\mathrm{P}}<0.05, \mathrm{DG}+\mathrm{A}$ vs. $\mathrm{DG}$.
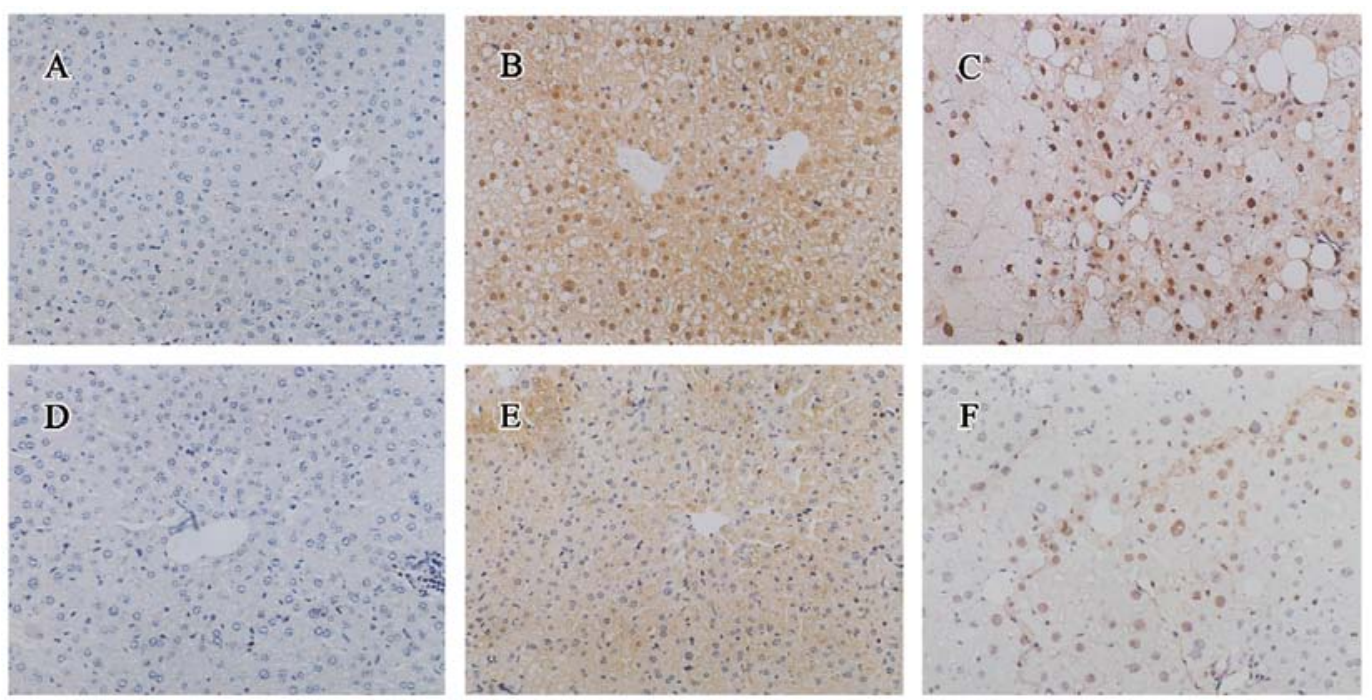

Figure 2. (A-C) Immunohistochemical analysis of tissue from the control group (CG), insulin resistance group (IG), and diabetes group (DG), respectively There was a significantly enhanced nuclear ubiquitin expression in the IG and DG along with different levels of tissue degeneratiaon (x200); (D-F) Control group treated with Astragalus polysaccharide (APS) (CG + A), insulin resistance group treated with APS (IG + A) and diabetes group treated with APS $(\mathrm{DG}+\mathrm{A})$, respectively. There was a significant improvement in nuclear ubiquitin expression in IG + A and DG + A compared with IG and DG (x200).
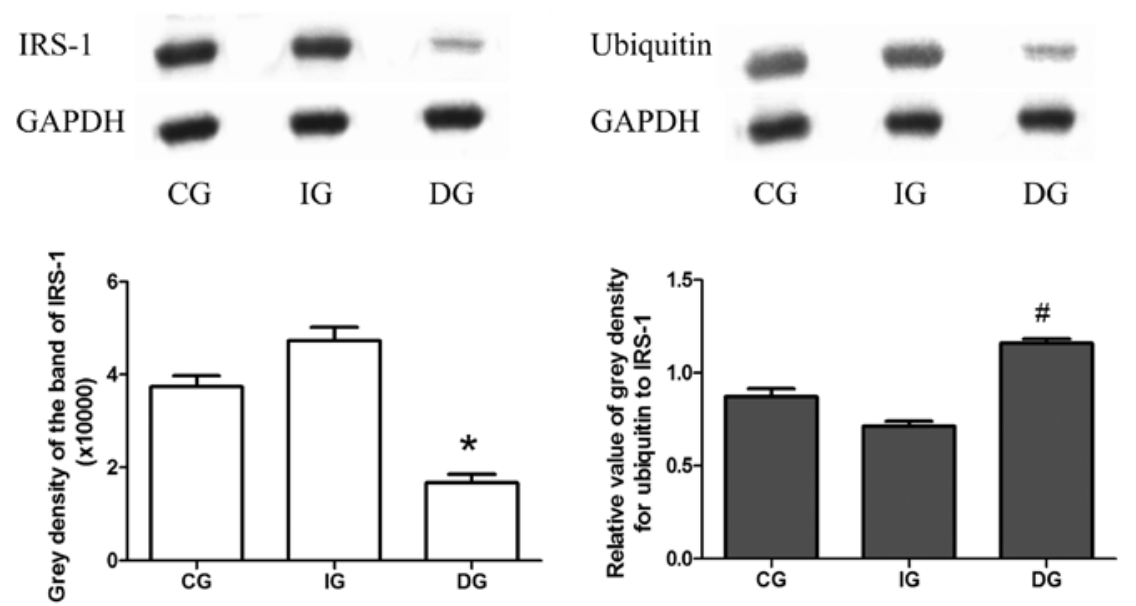

Figure 3. Western blot analysis of the expression of insulin receptor substrate-1 (IRS-1) and ubiquitin. Data were presented as the means \pm SEM. ${ }^{*} \mathrm{P}<0.05$, diabetes group (DG) vs. control group (CG) and insulin resistance group (IG); ${ }^{~} \mathrm{P}<0.05$, DG vs. IG.

\section{Effect of APS on diabetes}

Comparison of physiological and biochemical indexes among groups. Our data demonstrated that on the 20th week, APS significantly reduced the weight of the DG + A mcie compared with the DG mice $(\mathrm{P}<0.05)$ (Fig. 6A); FBG and PBG levels were significantly improved in the DG + A 

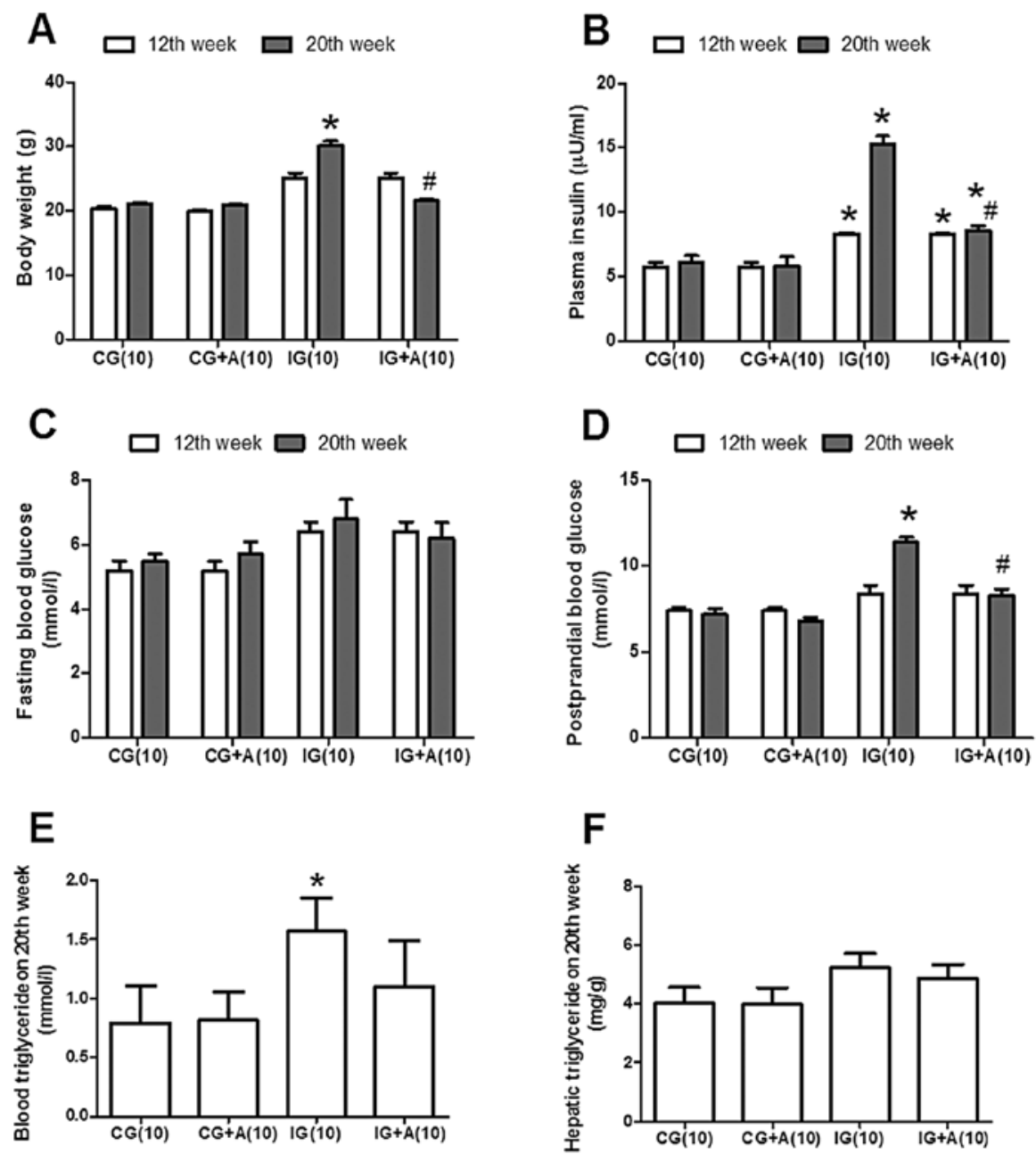

Figure 4. Effect of Astragalus polysaccharid(APS) on (A) body weight, (B) plasma insulin levels, (C) fasting blood glucose levels, (D) postprandial blood glucose levels, (E) blood triglyceride levels, and (F) hepatic triglyceride levels in the insulin resistance group. CG, control group; IG, insulin resistance group; CG + A, control group treated with Astragalus polysaccharide (APS); IG + A, insulin resistance group treated with APS. Data are presented as the means \pm SEM. Numbers in brackets denote the number of animals studied. ${ }^{*} \mathrm{P}<0.05$ or $0.01, \mathrm{CG}$ vs. the other groups on the same week; ${ }^{\sharp} \mathrm{P}<0.05$, IG vs. IG $+\mathrm{A}$ on the same week.
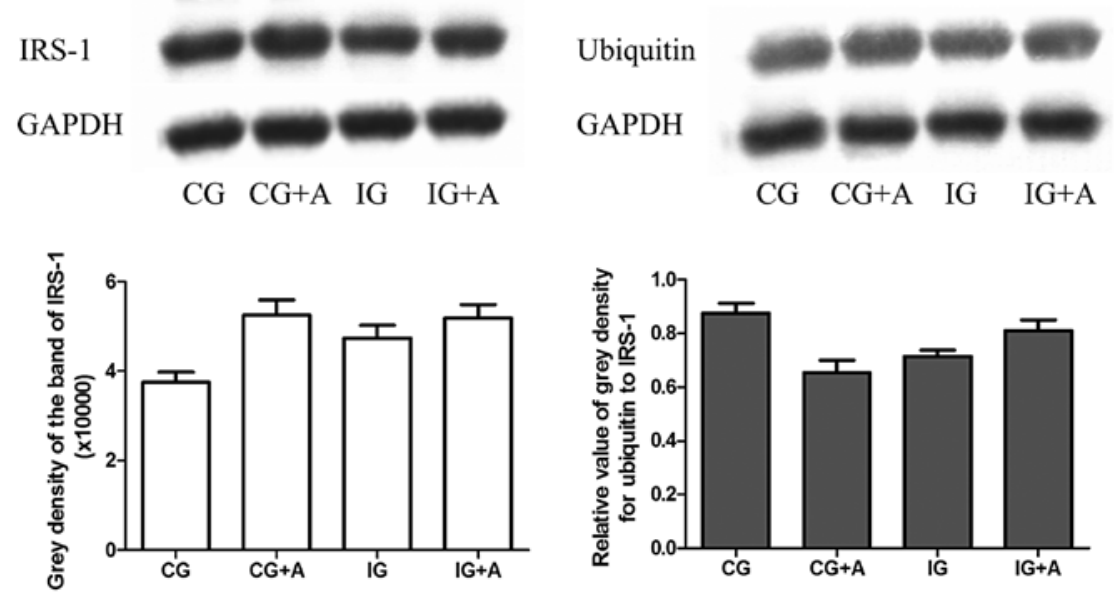

Figure 5. Western blot analysis of the expression of insulin receptor substrate-1 (IRS-1) and ubiquitin following treatment with Astragalus polysaccharide (APS) in the insulin resistance group. CG, control group; IG, insulin resistance group; CG + A, control group treated with APS; IG + A, insulin resistance group treated with APS. Data were presented as the means \pm SEM. There was no significant difference among the groups.

mice compared with the DG mice $(\mathrm{P}<0.05)$ (Fig. 6C and D); however, the plasma insulin levels were not significantly altered in the $\mathrm{DG}+\mathrm{A}$ mice $(\mathrm{P}>0.05)($ Fig. 6B). The triglyceride levels in the blood and liver were significantly improved in the 

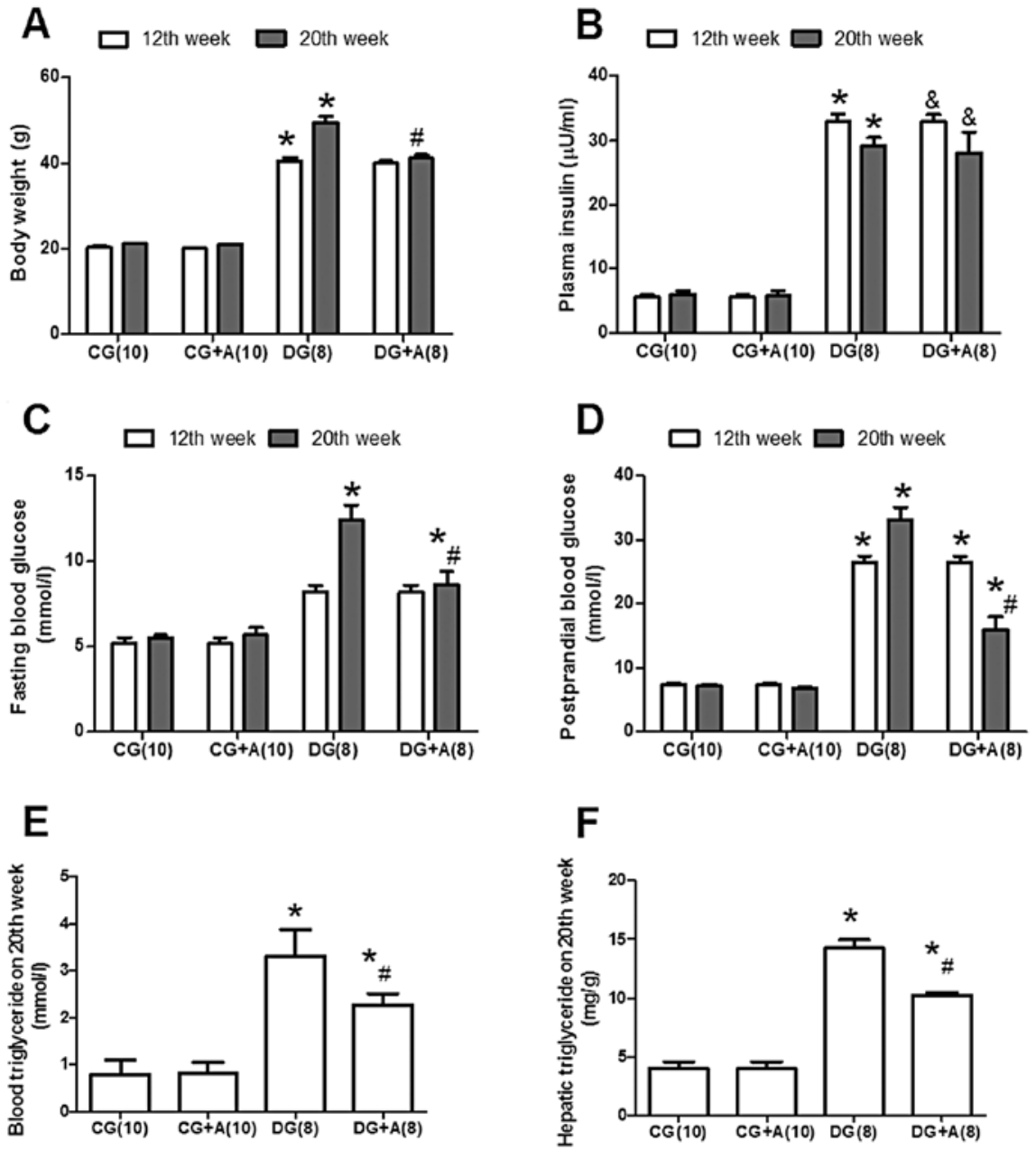

Figure 6. Effect of Astragalus polysaccharid(APS) on (A) body weight, (B) plasma insulin levels, (C) fasting blood glucose levels, (D) postprandial blood glucose levels, (E) blood triglyceride levels, and (F) hepatic triglyceride levels in the diabetes group. CG, control group; DG, diabetes group; CG + A, control group treated with APS; DG + A, diabetes group treated with APS. Data were presented as the means \pm SEM. Numbers in brackets denote the number of

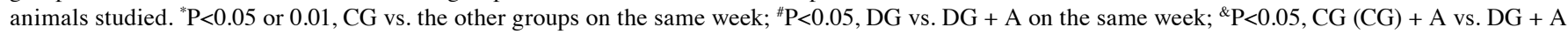
on the same week.

DG + A mice compared with the DG mice on the 20th week $(\mathrm{P}<0.05)$ (Fig. 6E and F).

Immunohistochemisty. As was observed under a light microscope, cellular edema and steatosis were markedly improved and the expression of ubiquitin in the nucleolus was partially reduced in DG + A mice compared with the DG mice (Fig. 2C and F). A comparison among the groups showed that the expression of ubiquitin in the nucleolus in the DG mice was significantly higher than that in the CG mice; however, this increase in expression was reversed by treatment with APS in the DG + A mice compared with the DG mice $(\mathrm{P}<0.05)$ (Table II).

Western blot analysis. The results from western blot analysis demonstrated that the expression of IRS-1 in the DG mice was significantly lower compared to that in the CG mice $(\mathrm{P}<0.05)$; although there was no significant difference in the expression of IRS- 1 between the DG and DG + A mice $(\mathrm{P}>0.05)$, treatment with APS increased the expression of IRS-1 to a certain extent and significantly downregulated the ubiquitination level of IRS-1 in the DG + A mice compared with the DG mice $(\mathrm{P}<0.05)$ (Fig. 7).

\section{Discussion}

Insulin signal transduction is a precise and sequentially controlled process. Any blocking or misregulation leads to the disruption of insulin signaling and the insensitivity of peripheral tissues to insulin stimulation, thus leading to insulin resistance. It is well known that insulin resistance combined with defective insulin secretion are the main reasons for the development of type 2 diabetes mellitus $(13,14)$. In this study, we established three groups representing the different characteristics of the main pathological stages for the development of type 2 diabetes mellitus and another three groups corresponding to treatment with APS in order to elucidate the underlying pathological mechanisms of type 2 diabetes from the perspective of ubiquitination, as well as the possible therapeutic effects and mechanisms of action of APS.

KKay mice originate from KK mice which were cultivated in 1941 by the Japanese scholar, K. Kondo using mice of the Kasukabe group. Toronto2(T2kk) mice, inbred mice, were cultivated by hybridization using the KK mice and C57BL/6J mice and reproduced by inbreeding. KK mice are the classic 


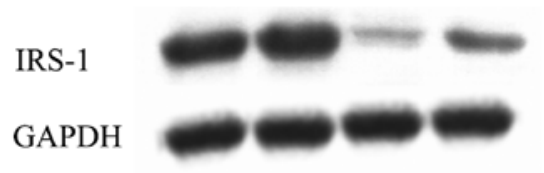

CG $\mathrm{CG}+\mathrm{A} \quad \mathrm{DG} \quad \mathrm{DG}+\mathrm{A}$

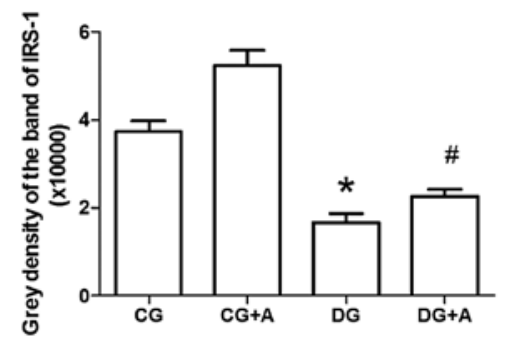

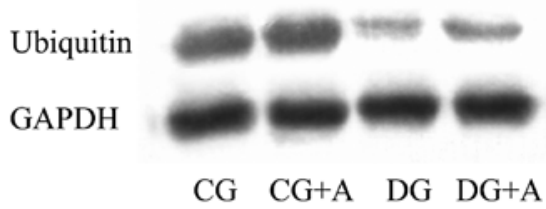

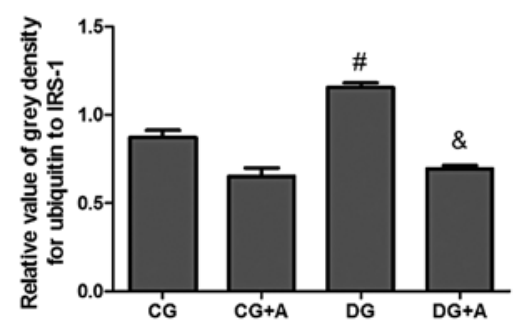

Figure 7. Western blot analysis of the expression of insulin receptor substrate-1 (IRS-1) and ubiquitin following treatment with Astragalus polysaccharide (APS) in the diabetes group Data are presented as the means \pm SEM. CG, control group; DG, diabetes group; CG + A, control group treated with APS; DG + A, diabetes group treated with APS. ${ }^{*} \mathrm{P}<0.05, \mathrm{CG}$ vs. $\mathrm{DG} ;{ }^{\prime \prime} \mathrm{P}<0.05, \mathrm{CG}+\mathrm{A}$ vs. the other groups; ${ }^{\&} \mathrm{P}<0.05, \mathrm{DG}+\mathrm{A}$ vs. DG.

polygenic animal model of diabetes with the classic characteristics of type 2 diabetes and have the characteristics of slight obesity, insulin resistance and hyperglycemia accompanied by a high incidence of type 2 diabetes. KKay mice are the mutants of the KK mice with the mutational color gene, which not only makes their coat hair yellow, but also causes metabolic disorders, such as obesity, hyperglycemia and hyperinsulinemia (15). As the development of type 2 diabetes in the KKay mice was induced by environmental factors on the basis of genetic susceptibility without any conscious artificial intervention, the symptoms and characteristics of KKay mice, such as hyperglycemia and insulin resistance were extremely similar to those of humans and this spontaneous animal model of type 2 diabetes was very suitable for the study of the pathological mechanisms and pharmacodynamics of diabetes; C57BL/6J mice, having good homology with KKay mice, were selected as the CG in this study.

To date, only the Gly->Arg972 substitution of IRS-1, interacting with environmental factors, seems to play a pathogenic role in the development of type 2 diabetes among the family members of IRS proteins (16). IRS-1, widely expressed in various types of peripheral tissue (liver, muscle and fat), serves as the docking protein which selectively docks with proteins containing Src-homology-2 (SH2) domains and regulates their activities or their subcellular location in order to maintain the follow-up insulin signal transduction process after the specific tyrosines of IRS-1 are phosphorylated by the $\beta$-subunits of the insulin receptor (17). IRS-1 acts as a signal amplifier by removing the stoichiometric constraints encountered by most receptors, directly recruiting $\mathrm{SH} 2$-proteins to their autophosphorylation sites (18). Glucose homeostasis is mainly maintained by IRS-1 and IRS-2 in different peripheral tissues, and both play complementary roles in insulin signaling and metabolism in the liver (19). It is certain that IRS-1 plays an indispensable rolem as it is highly expressed in the liver. However, there are some undiscovered roles of IRS-1 in the liver which account for the abnormal symptoms of type 2 diabetes and some undiscovered regulatory mechanisms which are worth investigating.
As shown in Fig. 1, it is evident that the DG mice showed the classic symptoms of type 2 diabetes, such as obesity, fasting hyperglycemia $(12.4 \pm 0.9 \mathrm{mmol} / \mathrm{l})$ and hyperinsulinemia $(29.2 \pm 1.2 \mu \mathrm{U} / \mathrm{ml})$ on the 20 th week, and the IG mice showed the classic characteristics of insulin resistance, such as hyperinsulinemia $(15.3 \pm 0.6 \mu \mathrm{U} / \mathrm{ml})$ with normal FBG levels $(6.8 \pm 0.6 \mathrm{mmol} / \mathrm{l})$. We also noticed that the IG mice had significant postprandial hyperglycemia and that the DG mice had both fasting hyperglycemia and postprandial hyperglycemia on the 20th week compared with the CG mice. As shown in Fig. 3, the expression of IRS-1 in the DG mice was significantly lower than that in the CG and IG mice; however, the ubiquitination level of IRS-1 in the DG mice was higher than that in the CG mice and significantly higher than that in the IG mice. These data indicated that postprandial hyperglycemia negatively correlated with the expression of IRS-1 in DG mice; this result was consistent with the results from a previous study on liver-specific IRS-1-knockout mice (20). These data also indicated that the ubiquitination of IRS-1 mainly contributed to the quantitative changes in the expression of IRS-1 in DG mice. Proteins tagged with ubiquitin usually trigger their degradation by the ubiquitin-proteasome system. The shortand long-term inhibition of the IRS protein function can be respectively attributed to serine phosphorylation and ubiquitintargeted proteosome-mediated degradation (2). According to these data, we hypothesized that the enhanced ubiquitination of IRS-1 was the underlying mechanism responsible for postprandial hyperglycemia in type 2 diabetes. As regards the fasting hyperglycemia in DG mice, we speculated that this abnormality may correlate with the abnormal expression or activity of IRS-2.

On the 20th week, both the IG and DG mice had gained significant amounts of weight compared with the CG mice and became obese (Fig. 1A). Obesity is the main cause of insulin resistance. Certain studies have revealed a significant reduction in the expression and tyrosine phosphorylation of IRS-1 in skeletal muscle from obese non-diabetic patients compared with healthy controls (21), and the expression of IRS-1 seems to be normal in non-obese diabetic patients accompanied by 
the reduced insulin-stimulated tyrosine phosphorylation of IRS-1 $(22,23)$. The results from this study revealed that the expression of IRS-1 in the livers of DG mice was markedly lower than that in CG mice. However, there was no significant change in IRS-1 expression and its ubiquitination level in the IG mice on the 20th week. The weight gain observed in the DG mice should partially correlate with the diminished expression of IRS-1 in the liver.

IRS-1-knockout mice exhibited hyperinsulinemia and resistance to the hypoglycemic function of insulin (3). As shown in Fig. 1B, we observed a significant increase in plasma insulin levels in both the IG and DG mice, and the expression of IRS-1 was significantly downregulated in the livers of DG mice. It has been reported that IRS-1/- mice display characteristics of syndrome $\mathrm{X}$, including hypertriglyceridemia, hypertension and impaired endothelium-dependent vascular relaxation (24). It seemed that the significantly increased levels of blood triglyceride in DG mice were partially attributed to the downregulation in the expression of IRS-1. In fact, triglyceride levels in the liver and muscle are in a state of constant turnover, and insulin bioactivity can be impaired by the intracellular metabolites of triglycerides and free fatty acids (FFA). In the liver, the knockdown of IRS-1 has been shown to lead to the upregulation of gluconeogenesis; by contrast, the knockdown of IRS-2 causes an upregulation in the levels of lipogenic enzymes, fatty acid synthase and hepatic lipid accumulation (25). As regards the significant increase in hepatic triglyceride levels in DG mice (Fig. 1F), we hypothesized that this increase correlated with the impaired expression of IRS-2 in the liver.

When analyzing the data shown in Fig. 6, we concluded that APS effectively reduced body weight, as well as FBG, PBG, blood triglyceride and hepatic triglyceride levels when comparing the DG mice with the DG + A mice. One important reason for these therapeutic effects of APS was the attenuation of the ubiquitination of IRS-1 without intervening with the total expression of IRS-1; another reason was the marked reduction in ubiquitination in the hepatic nucleolus in $\mathrm{DG}+\mathrm{A}$ mice.

Increasing evidence has indicated that the ubiquitinproteasome system participates in the regulation of insulin signaling (26-28), and there have been several studies published on the transcriptional and post-transcriptional regulation of this system in the nucleolus (29-31). In our study, the results from immunohistochemistry revealed that the overexpression of ubiquitin in the hepatic nucleolus significantly correlated with the onset of insulin resistance and type 2 diabetes, when comparing the DG and IG mice with the CG mice. The transcriptional or post-transcriptional regulation of the overexpressed nuclear ubiquitin in the liver and the corresponding molecular mechanisms were the reasons for the development of insulin resistance and type 2 diabetes. We also observed that there was a positive correlation between the overexpression of nuclear ubiquitin and hyperglycemia. On the 20th week, there were different levels of overexpression of nuclear ubiquitin in the IG mice with postprandial hyperglycemia and in DG mice with fasting hyperglycemia and postprandial hyperglycemia; however, this increase in nuclear ubiquitin expression was reversed in both the $\mathrm{IG}+\mathrm{A}$ and $\mathrm{DG}+\mathrm{A}$ mice, accompanied by significantly improved hyperglycemia compared with the corresponding untreated group. Apart from the involvement of IRS-1 in the regulation of glycometabolism, we speculated that significant changes in nuclear ubiquitin expression and the related ubiquitination pathway were important regulators for insulin signaling and glycometabolism. The other meaningful discovery was that the overexpression of ubiquitin in the hepatic nucleolus was markedly downregulated by APS in the $\mathrm{IG}+\mathrm{A}$ and $\mathrm{DG}+\mathrm{A}$ mice compared with the corresponding untreated group. As it is currently known, peroxisome proliferator-activated receptor $\gamma(\operatorname{PPAR} \gamma)$ in the nucleolus is the target protein of troglitazone, an insulin sensitizer; there are a number of publications on the ubiquitin-proteasome regulation of PPAR $\gamma$ (32-34). APS, exhibiting similar effects as an insulin sensitizer to troglitazone by the attenuation of the ubiquitination of IRS-1, may also play a profound regulatory role in the ubiquitination of PPAR $\gamma$ in the nucleolus. Further studies are required to investigate this issue to a greater extent.

\section{Ackowledgements}

We would like to express our gratitude to the cooperative partners from the Experimental Animal Center of Wuhan University and the Key Laboratory of Hubei Province for Digestive System Disease.

\section{References}

1. Boller S, Joblin BA, Xu L, et al: From signal transduction to signal interpretation: an alternative model for the molecular function of insulin receptor substrates. Arch Physiol Biochem 118: 148-155, 2012.

2. White MF: IRS proteins and the common path to diabetes. Am J Physiol Endocrinol Metab 283: E413-E422, 2002.

3. Tamemoto $\mathrm{H}$, Kadowaki $\mathrm{T}$, Tobe $\mathrm{K}$, et al: Insulin resistance and growth retardation in mice lacking insulin receptor substrate-1. Nature 372: 182-186, 1994.

4. Draznin B: Molecular mechanisms of insulin resistance: serine phosphorylation of insulin receptor substrate-1 and increased expression of p85alpha: the two sides of a coin. Diabetes 55: 2392-2397, 2006.

5. Gual P, Le Marchand-Brustel Y and Tanti JF: Positive and negative regulation of insulin signaling through IRS-1 phosphorylation. Biochimie 87: 99-109, 2005.

6. Lee AV, Gooch JL, Oesterreich S, Guler RL and Yee D: Insulin-like growth factor I-induced degradation of insulin receptor substrate 1 is mediated by the $26 \mathrm{~S}$ proteasome and blocked by phosphatidylinositol 3'-kinase inhibition. Mol Cell Biol 20: 1489-1496, 2000.

7. Chen W, Xia YP, Chen WJ, Yu MH, Li YM and Ye HY: Improvement of myocardial glycolipid metabolic disorder in diabetic hamster with Astragalus polysaccharides treatment. Mol Biol Rep 39: 7609-7615, 2012.

8. Wang N, Zhang D, Mao X, Zou F, Jin H and Ouyang J: Astragalus polysaccharides decreased the expression of PTP1B through relieving ER stress induced activation of ATF6 in a rat model of type 2 diabetes. Mol Cell Endocrinol 307: 89-98, 2009.

9. Kallon S, Li X, Ji J, et al: Astragalus polysaccharide enhances immunity and inhibits H9N2 avian influenza virus in vitro and in vivo. J Anim Sci Biotechnol 4: 22, 2013.

10. Lu J, Chen X, Zhang Y, et al: Astragalus polysaccharide induces anti-inflammatory effects dependent on AMPK activity in palmitate-treated RAW264.7 cells. Int J Mol Med 31: 1463-1470, 2013.

11. Wu Y, Ou-Yang JP, Wu K, Wang Y, Zhou YF and Wen CY: Hypoglycemic effect of Astragalus polysaccharide and its effect on PTP1B. Acta Pharmacol Sin 26: 345-352, 2005.

12. Mao XQ, Wu Y, Wu K, Liu M, Zhang JF, Zou F and Ou-Yang JP: Astragalus polysaccharide reduces hepatic endoplasmic reticulum stress and restores glucose homeostasis in a diabetic KKAy mouse model. Acta Pharmacol Sin 28: 1947-1956, 2007.

13. Saltiel AR and Kahn CR: Insulin signalling and the regulation of glucose and lipid metabolism. Nature 414: 799-806, 2001. 
14. Pessin JE and Saltiel AR: Signaling pathways in insulin action: molecular targets of insulin resistance. J Clin Invest 106: 165-169, 2000.

15. Iwatsuka H, Shino A and Suzuoki Z: General survey of diabetic features of yellow KK mice. Endocrinol Jpn 17: 23-35, 1970.

16. Hitman GA, Hawrami K, McCarthy MI, et al: Insulin receptor substrate-1 gene mutations in NIDDM; implications for the study of polygenic disease. Diabetologia 38: 481-486, 1995.

17. White MF: The IRS-signalling system: a network of docking proteins that mediate insulin action. Mol Cell Biochem 182: 3-11, 1998.

18. White MF: The IRS-signalling system in insulin and cytokine action. Philos Trans R Soc Lond B Biol Sci 351: 181-189, 1996.

19. Thirone AC, Huang $C$ and Klip A: Tissue-specific roles of IRS proteins in insulin signaling and glucose transport. Trends Endocrinol Metab 17: 72-78, 2006.

20. Kubota N, Kubota T, Itoh S, et al: Dynamic functional relay between insulin receptor substrate 1 and 2 in hepatic insulin signaling during fasting and feeding. Cell Metab 8: 49-64, 2008.

21. Goodyear LJ, Giorgino F, Sherman LA, Carey J, Smith RJ and Dohm GL: Insulin receptor phosphorylation, insulin receptor substrate-1 phosphorylation, and phosphatidylinositol 3-kinase activity are decreased in intact skeletal muscle strips from obese subjects. J Clin Invest 95: 2195-2204, 1995.

22. Björnholm M, Kawano Y, Lehtihet M and Zierath JR: Insulin receptor substrate-1 phosphorylation and phosphatidylinositol 3-kinase activity in skeletal muscle from NIDDM subjects after in vivo insulin stimulation. Diabetes 46: 524-527, 1997.

23. Krook A, Björnholm M, Galuska D, et al: Characterization of signal transduction and glucose transport in skeletal muscle from type 2 diabetic patients. Diabetes 49: 284-292, 2000.

24. Abe H, Yamada N, Kamata K, et al: Hypertension, hypertriglyceridemia, and impaired endothelium-dependent vascular relaxation in mice lacking insulin receptor substrate-1. J Clin Invest 101: 1784-1788, 1998.
25. Taniguchi CM, Ueki K and Kahn R: Complementary roles of IRS- 1 and IRS-2 in the hepatic regulation of metabolism. J Clin Invest 115: 718-727, 2005 .

26. Song R, Peng W, Zhang Y, et al: Central role of E3 ubiquitin ligase MG53 in insulin resistance and metabolic disorders. Nature 494: 375-379, 2013

27. Chen YC, Fueger PT and Wang Z: Depletion of PAK1 enhances ubiquitin-mediated survivin degradation in pancreatic $\beta$-cells. Islets 5: 22-28, 2013.

28. Kirk-Ballard H, Wang ZQ, Acharya P, et al: An extract of Artemisia dracunculus L. inhibits ubiquitin-proteasome activity and preserves skeletal muscle mass in a murine model of diabetes. PLoS One 8: e57112, 2013.

29. Conaway RC, Brower CS and Conaway JW: Emerging roles of ubiquitin in transcription regulation. Science 296: 1254-1258, 2002.

30. Muratani M and Tansey WP: How the ubiquitin-proteasome system controls transcription. Nat Rev Mol Cell Biol 4: 192-201, 2003.

31. Wadosky KM and Willis MS: The story so far: post-translational regulation of peroxisome proliferator-activated receptors by ubiquitination and SUMOylation. Am J Physiol Heart Circ Physiol 302: H515-H526, 2012.

32. Han L, Wang P, Zhao G, Wang H, Wang M, Chen J and Tong T: Upregulation of SIRT1 by $17 \beta$-estradiol depends on ubiquitinproteasome degradation of PPAR $-\gamma$ mediated by NEDD4-1. Protein Cell 4: 310-321, 2013.

33. Kilroy G, Kirk-Ballard H, Carter LE and Floyd ZE: The ubiquitin ligase Siah2 regulates PPAR $\gamma$ activity in adipocytes. Endocrinology 153: 1206-1218, 2012.

34. Stravodimou A, Mazzoccoli G and Voutsadakis IA: Peroxisome proliferator-activated receptor gamma and regulations by the ubiquitin-proteasome system in pancreatic cancer. PPAR Res 2012: 367450, 2012. 